\title{
Wanted: a reward for antibiotic development
}

\author{
Addressing the commercial failure of the antibiotic market should be a priority for governments seeking to encourage \\ development of new drugs against resistant bugs.
}

A

$t$ the end of May, the Nature conference "Countering Antimicrobial

Resistance" in Beijing, China, showcased a wide array of discovery approaches focused on combatting drug-resistant bacteria. On the one hand, the sheer diversity of candidate treatments on show-from novel antibiotics, antimicrobial peptides and peptide fusions, therapeutic antibodies, microbiome treatments, CRISPR anti-infectives to therapeutic vaccines-was encouraging. On the other, several speakers warned that few of these interventions are likely to reach patients unless more is done to address the continued market failure of antibiotics. In June, the US Food and Drug Administration laid out its agenda for addressing the problem of antibiotic reimbursement. But real progress in galvanizing product development will likely require governments and international agencies to undertake a coordinated, collective, global effort that offers industry tangible financial rewards for entering the marketplace.

According to the AMR Review, a 2016 UK government report on antimicrobial resistance (AMR) funded by the Wellcome Trust, at least 700,000 people die annually owing to drug-resistant pathogens. In recent years, 'superbugs', such as methicillin-resistant Staphylococcus aureus and extremely drug-resistant (XDR) Mycobacterium tuberculosis, have been joined in hospitals or community settings by Gram-negative organisms, such as carbapenem-resistant Acinetobacter baumannii and Pseudomonas aeruginosa. By 2050, based on the current rate of antibiotic approvals, the UK report estimates that drug-resistant superbugs could kill up to 10 million people.

Paradoxically, as this AMR problem grows, commercial incentives for industry to develop new antibiotics are diminishing.

At the Nature event, promising early clinical data was presented on a new monobactam derivative (LYS228) from Novartis; and yet the company is currently seeking to outlicense the asset. A similar story can be seen across big pharma; only Merck \& Co., Roche, GlaxoSmithKline and Pfizer now have active antibiotic programs, and only 12 antibiotics have been approved since 2000-a decision on Achaogen's plazomicin was expected as Nature Biotechnology went to press.

Manufacturers are deserting novel antibiotic development for several reasons. Recruiting patients for trials is both laborious and challenging because clinical centers move to quickly contain AMR outbreaks. Demonstrating clinical benefit in a reasonable timeframe is also challenging due to the sporadic nature of AMR and the difficulty of demonstrating the superiority of a novel agent over an existing, curative antibiotic. From a business standpoint, standard drug reimbursement, which links return on investment to the volume of drug sold, simply doesn't work for antibiotics.

Faced with a dwindling antibacterial pipeline, governments have scrambled to plough more funding into R\&D-so-called push incentives. For example, the European Joint Programming Initiative on Antimicrobial Resistance, the Innovative Medicine Initiative's (IMI) New Drugs for
Bad Bugs program, the Global Antibiotics Research and Development Partnership, Novo Holdings' REPAIR Impact Fund and the Combating Antibiotic Resistant Bacteria Biopharmaceutical Accelerator (CARB-X) initiative will collectively inject over \$1 billion between 2014 and 2021.

But these incentives are focused on drug R\&D rather than supporting the market after approval. No one has successfully deployed the missing piece to the puzzle: a strong market pull incentive.

Last month, FDA Commissioner Scott Gottlieb publicly acknowledged that the antibiotic market was broken, requiring a large pull incentive. $\mathrm{He}$ went on to propose a specific pull mechanism - a model in which acutecare institutions would pay a fixed licensing fee in return for access to a certain number of annual doses of a new antibiotic. Although it is encouraging that the agency is thinking about market incentives, negotiating contracts with thousands of medical centers, long-term care facilities and private payers (all currently incentivized to cut costs) seems rather impractical.

Other promising market incentives remain on the table, however.

DRIVE-AB, an IMI project specifically looking at the economics of antibiotic development, reviewed many pull mechanisms, including priority review vouchers and transfers of intellectual property rights (TIPR). The former have proven popular in neglected disease research, where a drug approval is awarded with a voucher that can be used to obtain priority review for another drug (such vouchers can be sold on to other manufacturers and priority review transferred). In contrast, TIPRs reward an approval with an exclusivity period (say 12 months) that is transferrable from the new antibiotic to another drug (e.g., a blockbuster), fending off generic competition.

In the final DRIVE-AB report, neither of these incentives won out. Instead, the 'market entry reward' (MER) was put forward. This is an award made to a drug developer in return for the approval of a novel antibiotic that meets a predefined target product profile (e.g., new target class, important medical need). DRIVE-AB estimates that a MER should be valued between $\$ 750$ million and $\$ 2,000$ million, paid out in installments over five years. And this concept may be reduced to practice. Already, the 2006 Pandemic and All Hazards Preparedness Act, which is being prepared for reauthorization by the US Congress, contains provisions that would give the Biomedical Advanced Research and Development Authority (BARDA) the ability to provide MERs for new antimicrobials addressing urgent unmet needs. Such MERs may be financed by TIPRs rather than general appropriations, giving companies more assurance of a reward once their drug passes the finish line in the years ahead.

That sounds like a step in the right direction. But AMR is global, not local. Europe and Asia must also embrace MER incentives if the range of antibiotic discovery research on display at the Nature Conference is to realize its potential. What is needed now is to convince industry that there are sufficient rewards to justify the considerable investment in developing these candidates into antimicrobial products of the future. 